\title{
FORCING WITH COPIES OF COUNTABLE ORDINALS
}

\author{
MILOŠ S. KURILIĆ
}

(Communicated by Mirna Džamonja)

\begin{abstract}
Let $\alpha$ be a countable ordinal and $\mathbb{P}(\alpha)$ the collection of its subsets isomorphic to $\alpha$. We show that the separative quotient of the poset $\langle\mathbb{P}(\alpha), \subset\rangle$ is isomorphic to a forcing product of iterated reduced products of Boolean algebras of the form $P\left(\omega^{\gamma}\right) / \mathcal{I}_{\omega \gamma}$, where $\gamma \in \operatorname{Lim} \cup\{1\}$ and $\mathcal{I}_{\omega \gamma}$ is the corresponding ordinal ideal. Moreover, the poset $\langle\mathbb{P}(\alpha), \subset\rangle$ is forcing equivalent to a two-step iteration of the form $(P(\omega) / \text { Fin })^{+} * \pi$, where $[\omega] \Vdash$ " $\pi$ is an $\omega_{1}$ closed separative pre-order" and, if $\mathfrak{h}=\omega_{1}$, to $(P(\omega) / \text { Fin })^{+}$. Also we analyze the quotients over ordinal ideals $P\left(\omega^{\delta}\right) / \mathcal{I}_{\omega^{\delta}}$ and the corresponding cardinal invariants $\mathfrak{h}_{\omega^{\delta}}$ and $\mathfrak{t}_{\omega^{\delta}}$.
\end{abstract}

\section{INTRODUCTION}

The posets of the form $\langle\mathbb{P}(\mathbb{X}), \subset\rangle$, where $\mathbb{X}$ is a relational structure and $\mathbb{P}(\mathbb{X})$ the set of (the domains of) its isomorphic substructures, were considered in [7], where a classification of the relations on countable sets related to the forcing-related properties of the corresponding posets of copies is described. So, defining two structures to be equivalent if the corresponding posets of copies produce the same generic extensions, we obtain a rough classification of structures which, in general, depends on the properties of the model of set theory in which we work.

For example, under $\mathrm{CH}$ all countable linear orders are partitioned in only two classes. Namely, by [6], CH implies that for a non-scattered countable linear order $L$ the poset $\langle\mathbb{P}(L), \subset\rangle$ is forcing equivalent to the iteration $\mathbb{S} *(P(\check{\omega}) / \text { Fin })^{+}$, where $\mathbb{S}$ is the Sacks forcing. Otherwise, for scattered orders, by [8] we have

Theorem 1.1. For each countable scattered linear order $L$ the separative quotient of the poset $\langle\mathbb{P}(L), \subset\rangle$ is $\omega_{1}$-closed and atomless. Under $C H$, it is forcing equivalent to the poset $(P(\omega) / \text { Fin })^{+}$.

The aim of this paper is to get a sharper picture of countable scattered linear orders in this context and we concentrate our attention on ordinals $\alpha<\omega_{1}$. So, in Section 3 we describe the separative quotient of the poset $\langle\mathbb{P}(\alpha), \subset\rangle$ and, in Section 5] factorize it as a two-step iteration $(P(\omega) / \text { Fin })^{+} * \pi$, where $[\omega] \Vdash$ " $\pi$ is an $\omega_{1}$-closed separative pre-order" (which implies that the equality $\mathfrak{h}=\omega_{1}$ implies that all posets

Received by the editors April 29, 2013 and, in revised form, September 6, 2013.

2010 Mathematics Subject Classification. Primary 03E40, 03E10, 03C15; Secondary 03E35, 03E17, 06A06.

This research was supported by the Ministry of Education and Science of the Republic of Serbia (Project 174006). 
$\langle\mathbb{P}(\alpha), \subset\rangle$ are forcing equivalent to $(P(\omega) / \text { Fin })^{+}$again $)$. In Section 4 we factorize the quotients $P\left(\omega^{\gamma}\right) / \mathcal{I}_{\omega^{\gamma}}$, for $\gamma \in \mathrm{Lim}$, and, in Section 6, consider the quotients over the ordinal ideals $P\left(\omega^{\delta}\right) / \mathcal{I}_{\omega^{\delta}}, 0<\delta<\omega_{1}$, and analyze the corresponding cardinal invariants $\mathfrak{h}_{\omega^{\delta}}$ and $\mathfrak{t}_{\omega^{\delta}}$.

We note that, while the results of the present paper are obtained using the Cantor normal form theorem for ordinals, the corresponding results concerning countable scattered linear orders given in 8 , are obtained from its analogue, Laver's theorem, stating that each countable scattered linear order is a finite sum of hereditarily additively indecomposable linear orders.

\section{Preliminaries}

In this section we recall some definitions and basic facts used in the paper.

If $\mathbb{X}$ is a relational structure, $X$ its domain and $A \subset X$, then $\mathbb{A}$ will denote the corresponding substructure of $\mathbb{X}$. Let $\mathbb{P}(\mathbb{X})=\{A \subset X: \mathbb{A} \cong \mathbb{X}\}$ and let $\mathcal{I}_{\mathbb{X}}=\{A \subset X: \mathbb{X} \nrightarrow \mathbb{A}\}$. It is easy to check that $\mathbb{X}$ is an indivisible structure (that is, for each partition $X=A \cup B$ we have $\mathbb{X} \hookrightarrow \mathbb{A}$, or $\mathbb{X} \hookrightarrow \mathbb{B})$ iff $\mathcal{I}_{\mathbb{X}}$ is an ideal. We will use the following elementary fact.

Fact 2.1. Let $\mathbb{X}$ and $\mathbb{Y}$ be relational structures and $f: \mathbb{X} \stackrel{\text { iso }}{\longrightarrow} \mathbb{Y}$. Then

(a) $A \in \mathcal{I}_{\mathbb{X}} \Leftrightarrow f[A] \in \mathcal{I}_{\mathbb{Y}}$, for each $A \subset X$;

(b) $\left\langle P(X) \backslash \mathcal{I}_{\mathbb{X}}, \subset\right\rangle \cong\left\langle P(Y) \backslash \mathcal{I}_{\mathbb{Y}}, \subset\right\rangle$.

A linear order $L$ is said to be scattered iff it does not contain a dense suborder or, equivalently, iff the rational line, $\mathbb{Q}$, does not embed in $L$. By $\mathcal{S}$ we denote the class of all countable scattered linear orders. A linear order $L$ is said to be additively indecomposable iff for each decomposition $L=L_{0}+L_{1}$ we have $L \hookrightarrow L_{0}$ or $L \hookrightarrow L_{1}$. The class $\mathcal{H}$ of hereditarily additively indecomposable (or ha-indecomposable) linear orders is the smallest class of order types of countable linear orders containing the one element order type, $\mathbf{1}$, and containing the $\omega$-sum, $\sum_{\omega} L_{i}$, and the $\omega^{*}$-sum, $\sum_{\omega^{*}} L_{i}$, for each sequence $\left\langle L_{i}: i \in \omega\right\rangle$ in $\mathcal{H}$ satisfying

$$
\forall i \in \omega\left|\left\{j \in \omega: L_{i} \hookrightarrow L_{j}\right\}\right|=\aleph_{0} .
$$

Fact 2.2 (Laver, [10]). $\mathcal{H} \subset \mathcal{S}$. If $L \in \mathcal{S}$, then $L \in \mathcal{H}$ iff $L$ is additively indecomposable (see also [11], p. 196 and p. 201).

Fact 2.3 (See [8]). (a) Let $L=\sum_{\omega} L_{i} \in \mathcal{H}$, where $\left\langle L_{i}: i \in \omega\right\rangle$ is a sequence in $\mathcal{H}$ satisfying (2.1). Then $A \subset L$ contains a copy of $L$ iff for each $i, m \in \omega$ there is finite $K \subset \omega \backslash m$ such that $L_{i} \hookrightarrow \bigcup_{j \in K} L_{j} \cap A$.

(b) Let $L=\sum_{i \leq n} L_{i}$, where $L_{i} \in \mathcal{H}$ are $\omega$-sums of sequences in $\mathcal{H}$ satisfying (2.1) and $L_{i}+L_{i+1} \notin \mathcal{H}$, for $i<n$. Then $\langle\mathbb{P}(L), \subset\rangle \cong \prod_{i \leq n}\left\langle\mathbb{P}\left(L_{i}\right), \subset\right\rangle$.

If $\langle A,<\rangle$ is a well ordering, type $\langle A,<\rangle$ denotes the unique ordinal isomorphic to $\langle A,<\rangle$. The product of ordinals $\alpha$ and $\beta$ is the ordinal $\alpha \beta=\operatorname{type}\langle\beta \times \alpha,<$ lex $\rangle$, where $<_{\operatorname{lex}}$ is the lexicographic order on the product $\beta \times \alpha$ defined by $\langle\xi, \zeta\rangle<_{\text {lex }}$ $\left\langle\xi^{\prime}, \zeta^{\prime}\right\rangle \Leftrightarrow \xi<\xi^{\prime} \vee\left(\xi=\xi^{\prime} \wedge \zeta<\zeta^{\prime}\right)$. The power $\alpha^{\beta}$ is defined recursively by $\alpha^{0}=1, \alpha^{\beta+1}=\alpha^{\beta} \alpha$ and $\alpha^{\gamma}=\sup \left\{\alpha^{\xi}: \xi<\gamma\right\}$, for limit $\gamma$. For an ordinal $\alpha$, instead of $\mathbb{P}(\langle\alpha, \in\rangle)$ we will write $\mathbb{P}(\alpha)$. 
Fact 2.4. For a countable limit ordinal $\alpha$ the following conditions are equivalent:

(a) $\alpha$ is indecomposable (i.e. $\alpha$ is not a sum of two smaller ordinals);

(b) $\beta+\gamma<\alpha$, for each $\beta, \gamma<\alpha$;

(c) $A \in \mathbb{P}(\alpha)$ or $\alpha \backslash A \in \mathbb{P}(\alpha)$, for each $A \subset \alpha$;

(d) $\alpha=\omega^{\delta}$, for some countable ordinal $\delta>0$;

(e) $\alpha \in \mathcal{H}$;

(f) $\alpha$ is an indivisible structure;

(g) $\mathcal{I}_{\alpha}=\{I \subset \alpha: \alpha \hookrightarrow I\}$ is an ideal in $P(\alpha)$.

Proof. For the equivalence of (b), (c) and (d) see [5], p. 43. For (a) $\Leftrightarrow$ (d) see 1.3 .6 of [2]. By [11, p. 176, (d) holds iff $\alpha$ is additively indecomposable which is, by Fact 2.2. equivalent to (e). (a) $\Leftrightarrow$ (f) is 6.8 .1 of [2]. (f) $\Leftrightarrow$ (g) is evident.

Fact 2.5. For each ordinal $\alpha$ we have $\mathbb{P}(\alpha)=P(\alpha) \backslash \mathcal{I}_{\alpha}$. Thus $\mathbb{P}\left(\omega^{\delta}\right)=\left(\mathcal{I}_{\omega^{\delta}}\right)^{+}$.

Proof. The inclusion " $\subset$ " is trivial. If $\alpha \hookrightarrow A \subset \alpha$, then, using the fact that for each increasing function $f: \alpha \rightarrow \alpha$ we have $\beta \leq f(\beta)$, for each $\beta \in \alpha$, we easily show that type $(A)=\alpha$, which means that $A \in \mathbb{P}(\alpha)$.

A partial order $\mathbb{P}=\langle P, \leq\rangle$ is called separative iff for each $p, q \in P$ satisfying $p \not \leq q$ there is $r \leq p$ such that $r \perp q$. The separative modification of $\mathbb{P}$ is the separative pre-order $\operatorname{sm}(\mathbb{P})=\left\langle P, \leq^{*}\right\rangle$, where $p \leq^{*} q \Leftrightarrow \forall r \leq p \exists s \leq r s \leq q$. The separative quotient of $\mathbb{P}$ is the separative partial order $\operatorname{sq}(\mathbb{P})=\left\langle P /=^{*}, \unlhd\right\rangle$, where $p={ }^{*} q \Leftrightarrow p \leq^{*} q \wedge q \leq^{*} p$ and $[p] \unlhd[q] \Leftrightarrow p \leq^{*} q$ (see [4]).

Fact 2.6. Let $\mathbb{P}, \mathbb{Q}$ and $\mathbb{P}_{i}, i \in I$, be partial orderings. Then

(a) $\mathbb{P}, \operatorname{sm}(\mathbb{P})$ and $s q(\mathbb{P})$ are forcing equivalent forcing notions;

(b) $\mathbb{P} \cong \mathbb{Q}$ implies that $\operatorname{sm} \mathbb{P} \cong \mathrm{sm} \mathbb{Q}$ and $\mathrm{sq} \mathbb{P} \cong \mathrm{sq} \mathbb{Q}$;

(c) $\operatorname{sm}\left(\prod_{i \in I} \mathbb{P}_{i}\right)=\prod_{i \in I} \operatorname{sm} \mathbb{P}_{i}$ and $\operatorname{sq}\left(\prod_{i \in I} \mathbb{P}_{i}\right) \cong \prod_{i \in I} \mathrm{sq} \mathbb{P}_{i}$.

Let $X$ be an infinite set, $\mathcal{I} \varsubsetneqq P(X)$ an ideal and $[X]^{<\omega} \subset \mathcal{I}$. Then

(d) $\operatorname{sm}\langle P(X) \backslash \mathcal{I}, \subset\rangle=\left\langle P(X) \backslash \mathcal{I}, \subset_{\mathcal{I}}\right\rangle$, where $A \subset_{\mathcal{I}} B \Leftrightarrow A \backslash B \in \mathcal{I}$.

(e) $\operatorname{sq}\langle P(X) \backslash \mathcal{I}, \subset\rangle=\left\langle\left(P(X) /={ }_{\mathcal{I}}\right)^{+}, \leq_{\mathcal{I}}\right\rangle$, where $A={ }_{\mathcal{I}} B \Leftrightarrow A \Delta B \in \mathcal{I}$ and $[A] \leq_{\mathcal{I}}[B] \Leftrightarrow A \backslash B \in \mathcal{I}$. Usually this poset is denoted by $(P(X) / \mathcal{I})^{+}$.

Let $\kappa$ be a regular cardinal. A pre-order $\langle\mathbb{P}, \leq\rangle$ is $\kappa$-closed iff for each $\gamma<\kappa$ and each sequence $\left\langle p_{\alpha}: \alpha<\gamma\right\rangle$ in $\mathbb{P}$, such that $\alpha<\beta \Rightarrow p_{\beta} \leq p_{\alpha}$, there is $p \in \mathbb{P}$ such that $p \leq p_{\alpha}$, for all $\alpha<\gamma$.

Fact 2.7. Let $\kappa$ be a regular cardinal and $\lambda$ an infinite cardinal. Then

(a) If $\mathbb{P}_{i}, i \in I$, are $\kappa$-closed pre-orders, then the product $\prod_{i \in I} \mathbb{P}_{i}$ is $\kappa$-closed.

(b) If $\mathfrak{c}=\omega_{1}$, then each atomless separative $\omega_{1}$-closed pre-order of size $\omega_{1}$ is forcing equivalent to $(P(\omega) / \mathrm{Fin})^{+}$(and to the collapsing algebra $\operatorname{Coll}\left(\omega_{1}, \omega_{1}\right)$ ).

(c) If $\lambda^{<\kappa}=\lambda$, then each atomless separative $\kappa$-closed pre-order $\mathbb{P}$ of size $\lambda$, such that $1_{\mathbb{P}} \Vdash|\check{\lambda}|=\check{\kappa}$, is forcing equivalent to the collapsing algebra $\operatorname{Coll}(\kappa, \lambda)$.

\section{The SeParative quotient of $\langle\mathbb{P}(\alpha) \subset\rangle$}

For a Boolean lattice $\mathbb{B}=\langle B, \leq\rangle$, by $\operatorname{rp}(\mathbb{B})$ we will denote the reduced power $\left\langle B^{\omega} / \equiv, \leq \equiv\right\rangle$, where for $\left\langle b_{i}\right\rangle,\left\langle c_{i}\right\rangle \in B^{\omega},\left\langle b_{i}\right\rangle \equiv\left\langle c_{i}\right\rangle$ (resp. $\left.\left[\left\langle b_{i}\right\rangle\right]_{\equiv} \leq \equiv\left[\left\langle c_{i}\right\rangle\right]_{\equiv}\right)$ iff $b_{i}=c_{i}$ (resp. $b_{i} \leq c_{i}$ ), for all but finitely many $i \in \omega$. For $n \in \omega$ we define the set $\operatorname{rp}^{n}(\mathbb{B})$ by: $\operatorname{rp}^{0}(\mathbb{B})=\mathbb{B}$ and $\operatorname{rp}^{n+1}(\mathbb{B})=\operatorname{rp}\left(\operatorname{rp}^{n}(\mathbb{B})\right)$. 
The aim of this section is to prove the following statement.

Theorem 3.1. If $\alpha=\omega^{\gamma_{n}+r_{n}} s_{n}+\cdots+\omega^{\gamma_{0}+r_{0}} s_{0}+k$ is a countable ordinal presented in the Cantor normal form, where $k \in \omega, r_{i} \in \omega, s_{i} \in \mathbb{N}, \gamma_{i} \in \operatorname{Lim} \cup\{1\}$ and $\gamma_{n}+r_{n}>\cdots>\gamma_{0}+r_{0}$, then

$$
\mathrm{sq}\langle\mathbb{P}(\alpha), \subset\rangle \cong \prod_{i=0}^{n}\left(\left(\operatorname{rp}^{r_{i}}\left(P\left(\omega^{\gamma_{i}}\right) / \mathcal{I}_{\omega \gamma_{i}}\right)\right)^{+}\right)^{s_{i}} .
$$

A proof of Theorem 3.1 is given at the end of the section.

We remind the reader that, if $\mathcal{I}$ and $\mathcal{J}$ are ideals on the sets $X$ and $Y$ respectively, then their Fubini product $\mathcal{I} \times \mathcal{J}$ is the ideal on the set $X \times Y$ defined by $\mathcal{I} \times \mathcal{J}=$ $\left\{A \subset X \times Y:\left\{x \in X: \pi_{Y}[A \cap(\{x\} \times Y)] \in \mathcal{J}^{+}\right\} \in \mathcal{I}\right\}$, where $\pi_{Y}: X \times Y \rightarrow Y$ is the projection. In particular, if $X=\omega, \mathcal{I}=$ Fin and $L_{i}=\{i\} \times Y$, for $i \in \omega$, then for $A \subset \omega \times Y$ we have

$$
A \in \operatorname{Fin} \times \mathcal{J} \Leftrightarrow \exists j \in \omega \forall i \geq j \pi_{Y}\left[A \cap L_{i}\right] \in \mathcal{J} .
$$

For convenience let us define the sets $\omega^{n} \times Y, n \in \omega$, recursively by $\omega^{0} \times Y=Y$ and $\omega^{n+1} \times Y=\omega \times\left(\omega^{n} \times Y\right)$. Also we define the ideal $\operatorname{Fin}^{n} \times \mathcal{J}$ on the set $\omega^{n} \times Y$ by: $\operatorname{Fin}^{0} \times \mathcal{J}=\mathcal{J}$ and $\operatorname{Fin}^{n+1} \times \mathcal{J}=\operatorname{Fin} \times\left(\operatorname{Fin}^{n} \times \mathcal{J}\right)$. Some parts of the following lemma are folklore but, for completeness, we include their proofs.

Lemma 3.2. For each ordinal $1 \leq \beta<\omega_{1}$ and each $n \in \omega$ we have:
(a) $\left\langle\mathbb{P}\left(\omega^{\beta+n}\right), \subset\right\rangle \cong\left\langle P\left(\omega^{n} \times \omega^{\beta}\right) \backslash\left(\operatorname{Fin}^{n} \times \mathcal{I}_{\omega^{\beta}}\right), \subset\right\rangle$;
(b) $\mathcal{I}_{\omega^{\beta+n}} \cong \operatorname{Fin}^{n} \times \mathcal{I}_{\omega^{\beta}}$;
(c) $\operatorname{sq}\left\langle\mathbb{P}\left(\omega^{\beta+n}\right), \subset\right\rangle \cong\left(P\left(\omega^{n} \times \omega^{\beta}\right) /\left(\operatorname{Fin}^{n} \times \mathcal{I}_{\omega^{\beta}}\right)\right)^{+}$;
(d) $P\left(\omega^{n} \times \omega^{\beta}\right) /\left(\operatorname{Fin}^{n} \times \mathcal{I}_{\omega^{\beta}}\right) \cong \operatorname{rp}^{n}\left(P\left(\omega^{\beta}\right) / \mathcal{I}_{\omega^{\beta}}\right)$;
(e) $\operatorname{sq}\left(\mathbb{P}\left(\omega^{\beta+n}\right), \subset\right) \cong\left(\operatorname{rp}^{n}\left(P\left(\omega^{\beta}\right) / \mathcal{I}_{\omega^{\beta}}\right)\right)^{+}$.

Proof. For $n=0$ the statement follows from Fact 2.5. So, in the sequel we prove the statement for $n \in \mathbb{N}$.

Using induction we prove (a) and (b) simultaneously. First we show that

$$
\left\langle\mathbb{P}\left(\omega^{\beta+1}\right), \subset\right\rangle \cong\left\langle\left(\operatorname{Fin} \times \mathcal{I}_{\omega^{\beta}}\right)^{+}, \subset\right\rangle .
$$

By the properties of ordinal multiplication and exponentiation we have $\left\langle\omega^{\beta+1}, \in\right\rangle$ $=\left\langle\omega^{\beta} \omega, \in\right\rangle \cong\left\langle\omega \times \omega^{\beta},<_{\text {lex }}\right\rangle=\mathbb{L}$, where $\mathbb{L}=\sum_{i \in \omega} \mathbb{L}_{i}$ and, for $i \in \omega, \mathbb{L}_{i}=\left\langle L_{i},<_{i}\right\rangle$, $L_{i}=\{i\} \times \omega^{\beta}$ and $\langle i, \xi\rangle<_{i}\langle i, \zeta\rangle \Leftrightarrow \xi \in \zeta$, for $\xi, \zeta \in \omega^{\beta}$. So, for the function $f_{i}: L_{i} \rightarrow \omega^{\beta}$ defined by $f_{i}(\langle i, \xi\rangle)=\xi$ we have

$$
f_{i}=\pi_{\omega^{\beta}} \mid L_{i}:\left\langle L_{i},<_{i}\right\rangle \stackrel{\text { iso }}{\longrightarrow}\left\langle\omega^{\beta}, \in\right\rangle .
$$

Since $\left\langle\omega^{\beta+1}, \in\right\rangle \cong \mathbb{L}$, by Facts 2.5 and 2.1 (b) $\left\langle\mathbb{P}\left(\omega^{\beta+1}\right), \subset\right\rangle=\left\langle P\left(\omega^{\beta+1}\right) \backslash \mathcal{I}_{\omega^{\beta+1}}, \subset\right\rangle$ $\cong\left\langle P(\mathbb{L}) \backslash \mathcal{I}_{\mathbb{L}}, \subset\right\rangle$ so it remains to be shown that

$$
\mathcal{I}_{\mathbb{L}}^{+}=\left(\operatorname{Fin} \times \mathcal{I}_{\omega^{\beta}}\right)^{+} .
$$

Claim 3.3. For each $A \subset \omega \times \omega^{\beta}$ we have

(i) $A \in \mathcal{I}_{\mathbb{L}}^{+} \Leftrightarrow \forall j \in \omega \quad \exists K \in[\omega \backslash j]^{<\omega} \omega^{\beta} \hookrightarrow \bigcup_{i \in K} L_{i} \cap A$;

(ii) $A \in\left(\operatorname{Fin} \times \mathcal{I}_{\omega^{\beta}}\right)^{+} \Leftrightarrow \forall j \in \omega \quad \exists i \geq j \quad \omega^{\beta} \hookrightarrow L_{i} \cap A$.

Proof. (i) By (3.3), for each $i \in \omega$ we have $\mathbb{L}_{i} \cong \omega^{\beta}$ so, by Fact 2.4 we have $\mathbb{L}_{i} \in \mathcal{H}$ and, clearly, condition (2.1) is satisfied. By Fact 2.3. (a) we have $A \in \mathcal{I}_{\mathbb{L}}^{+}$iff $\forall j \in \omega \exists K \in[\omega \backslash j]^{<\omega} \omega^{\beta} \hookrightarrow \bigcup_{i \in K} L_{i} \cap A$. 
(ii) By (3.1), $A \notin$ Fin $\times \mathcal{I}_{\omega^{\beta}}$ iff for each $j \in \omega$ there exists $i \geq j$ such that $\pi_{\omega^{\beta}}\left[L_{i} \cap A\right] \notin \mathcal{I}_{\omega^{\beta}}$. But, by (3.3) and Fact 2.1(a) we have: $\pi_{\omega^{\beta}}\left[L_{i} \cap A\right] \notin \mathcal{I}_{\omega^{\beta}}$ iff $f_{i}\left[L_{i} \cap A\right] \notin \mathcal{I}_{\omega^{\beta}}$ iff $L_{i} \cap A \notin \mathcal{I}_{\mathbb{L}_{i}}$ iff $\mathbb{L}_{i} \hookrightarrow L_{i} \cap A$ iff $\omega^{\beta} \hookrightarrow L_{i} \cap A$.

By Claim 3.3, the inclusion " $\supset$ " in (3.4) is satisfied and we prove " $\subset$ ". If $A \in \mathcal{I}_{\mathbb{L}}^{+}$ and $j \in \omega$, then, by Claim 3.3 (i), there are $K \in[\omega \backslash j]^{<\omega}$ and $g: \omega^{\beta} \hookrightarrow \bigcup_{i \in K} L_{i} \cap A$. Let $i_{0}=\max \left\{i \in K: g\left[\omega^{\beta}\right] \cap L_{i} \cap A \neq \emptyset\right\}$. Then $F=g\left[\omega^{\beta}\right] \cap L_{i_{0}} \cap A$ is a final part of the linear order $g\left[\omega^{\beta}\right] \cong \omega^{\beta}$ and, since type $\left(g\left[\omega^{\beta}\right] \backslash F\right)<\omega^{\beta}$, by Fact 2.4 (c) we have type $(F)=\omega^{\beta}$ and, hence $\omega^{\beta} \hookrightarrow L_{i_{0}} \cap A$ and $i_{0} \geq j$. By (ii) of Claim 3.3 we have $A \in\left(\text { Fin } \times \mathcal{I}_{\omega^{\beta}}\right)^{+}$and (3.4) is proved. So (3.2) is true.

By (3.4) we have $\mathcal{I}_{\mathbb{L}}=$ Fin $\times \mathcal{I}_{\omega^{\beta}}$. Since $\left\langle\omega^{\beta+1}, \in\right\rangle \cong \mathbb{L}$, by Fact 2.1(a) we have $\mathcal{I}_{\omega^{\beta+1}} \cong \mathcal{I}_{\mathbb{L}}$ and, hence,

$$
\mathcal{I}_{\omega^{\beta+1}} \cong \operatorname{Fin} \times \mathcal{I}_{\omega^{\beta}} .
$$

Let us assume that the statements (a) and (b) are true for $n$. By (3.5) we have $\mathcal{I}_{\omega^{\beta+n+1}} \cong \operatorname{Fin} \times \mathcal{I}_{\omega^{\beta+n}} \cong \operatorname{Fin} \times\left(\operatorname{Fin}^{n} \times \mathcal{I}_{\omega^{\beta}}\right)=\operatorname{Fin}^{n+1} \times \mathcal{I}_{\omega^{\beta}}$. By Fact 2.5 we have $\left\langle\mathbb{P}\left(\omega^{\beta+n+1}\right), \subset\right\rangle=\left\langle\left(\mathcal{I}_{\omega^{\beta+n+1}}\right)^{+}, \subset\right\rangle \cong\left\langle\left(\operatorname{Fin}^{n+1} \times \mathcal{I}_{\omega^{\beta}}\right)^{+}, \subset\right\rangle$.

(c) follows from (a) and Fact 2.6 (b) and (e).

(d) We use induction. For a proof of (d) for $n=1$ we show that the mapping $F:\left\langle P\left(\omega \times \omega^{\beta}\right) /={ }_{\text {Fin }} \times \mathcal{I}_{\omega \beta}, \leq_{\text {Fin }} \times \mathcal{I}_{\omega \beta}\right\rangle \rightarrow\left\langle\left\langle P\left(\omega^{\beta}\right) /=_{\mathcal{I}_{\omega \beta}}, \leq_{\mathcal{I}_{\omega \beta}}\right\rangle^{\omega} / \equiv, \leq \equiv\right\rangle$, given

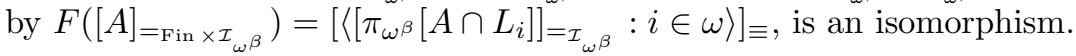

Claim 3.4. For $A, B \subset \omega \times \omega^{\beta}$ we have: $A={ }_{\mathrm{Fin} \times \mathcal{I}_{\omega \beta}} B$ if and only if

$$
\left[\left\langle\left[\pi_{\omega^{\beta}}\left[A \cap L_{i}\right]\right]_{=_{\mathcal{I}^{\beta} \beta}}: i \in \omega\right\rangle\right]_{\equiv}=\left[\left\langle\left[\pi_{\omega^{\beta}}\left[B \cap L_{i}\right]\right]_{=_{\mathcal{I}^{\beta}}}: i \in \omega\right\rangle\right]_{\equiv} .
$$

Proof. First, by (3.1) we have

$$
A={ }_{\text {Fin } \times \mathcal{I}_{\omega^{\beta}}} B \Leftrightarrow \exists j \in \omega \quad \forall i \geq j \pi_{\omega^{\beta}}\left[(A \triangle B) \cap L_{i}\right] \in \mathcal{I}_{\omega^{\beta}} .
$$

On the other hand, (3.6) holds iff there is $j \in \omega$ such that for all $i \geq j$ we have $\pi_{\omega^{\beta}}\left[A \cap L_{i}\right] \triangle \pi_{\omega^{\beta}}\left[B \cap L_{i}\right] \in \mathcal{I}_{\omega^{\beta}}$, that is, since the restriction $\pi_{\omega^{\beta}} \mid L_{i}$ is a bijection, $\left.\left(\pi_{\omega^{\beta}} \mid L_{i}\right)\left[\left(A \cap L_{i}\right) \triangle\left(B \cap L_{i}\right)\right]=\pi_{\omega^{\beta}}\left[(A \triangle B) \cap L_{i}\right)\right] \in \mathcal{I}_{\omega^{\beta}}$.

By Claim 3.4, $F$ is a well-defined injection.

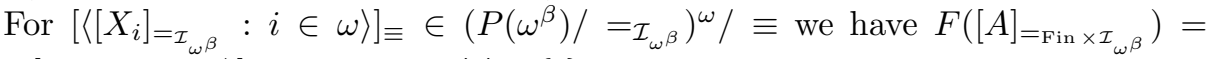
$\left[\left\langle\left[X_{i}\right]_{=_{\mathcal{I} \beta}}: i \in \omega\right\rangle\right]_{\equiv}$, where $A=\bigcup_{i \in I}\{i\} \times X_{i}$, so $F$ is a surjection.

By (3.1) we have $[A]_{=_{\text {Fin } \times \mathcal{I}_{\omega \beta}}} \leq_{\text {Fin } \times \mathcal{I}_{\omega \beta}}[B]_{=_{\text {Fin } \times \mathcal{I}_{\omega \beta}}}$ iff

$$
\exists j \in \omega \forall i \geq j \pi_{\omega^{\beta}}\left[A \backslash B \cap L_{i}\right] \in \mathcal{I}_{\omega^{\beta}}
$$

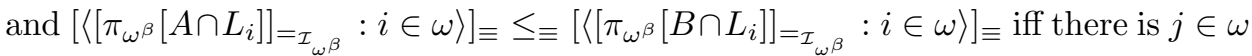
such that for all $i \geq j$ we have $\pi_{\omega^{\beta}}\left[A \cap L_{i}\right] \backslash \pi_{\omega^{\beta}}\left[B \cap L_{i}\right] \in \mathcal{I}_{\omega^{\beta}}$, that is, since the restriction $\pi_{\omega^{\beta}} \mid L_{i}$ is a bijection, $\left.\left(\pi_{\omega^{\beta}} \mid L_{i}\right)\left[\left(A \cap L_{i}\right) \backslash\left(B \cap L_{i}\right)\right]=\pi_{\omega^{\beta}}\left[A \backslash B \cap L_{i}\right)\right] \in$ $\mathcal{I}_{\omega^{\beta}}$. Thus $F$ is an isomorphism.

Assuming that the statement is true for $n$, by (b) and (d) for $n=1$ we have $P\left(\omega^{n+1} \times \omega^{\beta}\right) /\left(\operatorname{Fin}^{n+1} \times \mathcal{I}_{\omega^{\beta}}\right) \cong P\left(\omega \times\left(\omega^{n} \times \omega^{\beta}\right)\right) /\left(\operatorname{Fin} \times\left(\operatorname{Fin}^{n} \times \mathcal{I}_{\omega^{\beta}}\right)\right) \cong$ $\left.P\left(\omega \times \omega^{\beta+n}\right) /\left(\operatorname{Fin} \times \mathcal{I}_{\omega^{\beta+n}}\right)\right) \cong \operatorname{rp}\left(P\left(\omega^{\beta+n}\right) / \mathcal{I}_{\omega^{\beta+n}}\right) \cong \operatorname{rp}\left(P\left(\omega^{n} \times \omega^{\beta}\right) /\left(\operatorname{Fin}^{n} \times \mathcal{I}_{\omega^{\beta}}\right) \cong\right.$ $\operatorname{rp}\left(\operatorname{rp}^{n}\left(P\left(\omega^{\beta}\right) / \mathcal{I}_{\omega^{\beta}}\right)\right) \cong \operatorname{rp}^{n+1}\left(P\left(\omega^{\beta}\right) / \mathcal{I}_{\omega^{\beta}}\right)$.

(e) follows from $(\mathrm{c})$ and $(\mathrm{d})$.

For $n \in \mathbb{N}$, let the ideal Fin $^{n}$ on the set $\omega^{n}=\omega \times(\omega \times \cdots \times(\omega \times \omega) \ldots)(n$-many factors $)$ be defined by: $\operatorname{Fin}^{n}=\operatorname{Fin} \times($ Fin $\times \cdots \times($ Fin $\times$ Fin $) \ldots)(n$-many factors $)$. 
Then, by Lemma 3.2 we have

Corollary 3.5. For each $n \in \mathbb{N}$ we have:

(a) $\left\langle\mathbb{P}\left(\omega^{n}\right), \subset\right\rangle \cong\left\langle P\left(\omega^{n}\right) \backslash \mathrm{Fin}^{n}, \subset\right\rangle$ and $\mathcal{I}_{\omega^{n}} \cong \operatorname{Fin}^{n}$;

(b) $\operatorname{sq}\left(\mathbb{P}\left(\omega^{n}\right), \subset\right) \cong\left(\operatorname{rp}^{n-1}(P(\omega) / \text { Fin })\right)^{+}$.

Lemma 3.6. $\langle\mathbb{P}(\gamma+k), \subset\rangle \cong\langle\mathbb{P}(\gamma), \subset\rangle$, for each limit ordinal $\gamma$ and each $k \in \mathbb{N}$.

Proof. First we prove $\mathbb{P}(\gamma+k)=\{C \cup\{\gamma, \gamma+1, \ldots, \gamma+k-1\}: C \in \mathbb{P}(\gamma)\}$. The inclusion " $\supset$ " is evident. If $A \in \mathbb{P}(\gamma+k)$ and $f: \gamma+k \hookrightarrow \gamma+k$, where $A=f[\gamma+k]$, then, since $f$ is an increasing function, we have $f(\beta) \geq \beta$, for each $\beta \in \gamma+k$, which implies $f(\gamma+i)=\gamma+i$, for $i<k$, and, hence, $C=f[\gamma] \in \mathbb{P}(\gamma)$ and $A=C \cup\{\gamma, \gamma+1, \ldots, \gamma+k-1\}$.

Now it is easy to show that the mapping $F:\langle\mathbb{P}(\gamma), \subset\rangle \rightarrow\langle\mathbb{P}(\gamma+k), \subset\rangle$, given by $F(C)=C \cup\{\gamma, \gamma+1, \ldots, \gamma+k-1\}$, is an isomorphism.

Lemma 3.7. Let $\delta, \delta^{\prime}>0$ be countable ordinals. Then

(a) The ordinal $\omega^{\delta}$ is an $\omega$-sum of elements of $\mathcal{H}$ satisfying (2.1);

(b) $\delta \geq \delta^{\prime} \Rightarrow \omega^{\delta}+\omega^{\delta^{\prime}} \notin \mathcal{H}$.

Proof. (a) By Fact 2.4 we have $\omega^{\delta} \in \mathcal{H}$ and $\omega^{\delta}$ cannot be an $\omega^{*}$-sum (since it is a well ordering) so it is an $\omega$-sum of elements of $\mathcal{H}$ satisfying (2.1).

(b) Suppose that $\omega^{\delta}+\omega^{\delta^{\prime}} \in \mathcal{H}$. Then, by Fact 2.4, $\omega^{\delta}+\omega^{\delta^{\prime}}=\omega^{\delta^{\prime \prime}}$, for some ordinal $\delta^{\prime \prime}$ and, clearly $\omega^{\delta} \leq \omega^{\delta^{\prime \prime}}$. Now, $\omega^{\delta}=\omega^{\delta^{\prime \prime}}$ is impossible, since $\omega^{\delta}$ cannot be isomorphic to its proper initial segment and, hence, $\omega^{\delta}<\omega^{\delta^{\prime \prime}}$, which implies that $\omega^{\delta^{\prime}}<\omega^{\delta^{\prime \prime}}$ as well. But this is impossible by Fact 2.4 (b).

Proof of Theorem 3.1. By Lemma 3.6 we can assume that $k=0$. So, we have $\alpha=$ $\omega^{\gamma_{n}+r_{n}}+\cdots+\omega^{\gamma_{n}+r_{n}}+\cdots+\omega^{\gamma_{0}+r_{0}}+\cdots+\omega^{\gamma_{0}+r_{0}}=\sum_{j<\sum_{i=0}^{n} s_{i}} L_{j}$. By Lemma 3.7(a) for each $j$ the order $L_{j} \in \mathcal{H}$ and it is an $\omega$-sum of elements of $\mathcal{H}$ satisfying (2.1). By Lemma 3.7(b) $L_{j}+L_{j+1} \notin \mathcal{H}$ so, by Fact 2.3(b), $\langle\mathbb{P}(\alpha), \subset\rangle \cong \prod_{j<\sum_{i=0}^{n} s_{i}}\left\langle\mathbb{P}\left(L_{j}\right), \subset\right\rangle$ $=\prod_{i=0}^{n}\left\langle\mathbb{P}\left(\omega^{\gamma_{i}+r_{i}}\right), \subset\right\rangle^{s_{i}}$, which, together with Fact 2.6 (b), (c) and Lemma 3.2 (e), gives $\operatorname{sq}\langle\mathbb{P}(\alpha), \subset\rangle \cong \operatorname{sq} \prod_{i=0}^{n}\left\langle\mathbb{P}\left(\omega^{\gamma_{i}+r_{i}}\right), \subset\right\rangle^{s_{i}} \cong \prod_{i=0}^{n}\left(\mathrm{sq}\left\langle\mathbb{P}\left(\omega^{\gamma_{i}+r_{i}}\right), \subset\right\rangle\right)^{s_{i}} \cong$ $\prod_{i=0}^{n}\left(\left(\operatorname{rp}^{r_{i}}\left(P\left(\omega^{\gamma_{i}}\right) / \mathcal{I}_{\omega \gamma_{i}}\right)\right)^{+}\right)^{s_{i}}$.

Corollary 3.8. $\mathrm{sq}\langle\mathbb{P}(\omega n), \subset\rangle \cong\left((P(\omega) / \text { Fin })^{+}\right)^{n}$, for each $n \in \mathbb{N}$.

\section{Forcing With The QUotient $P\left(\omega^{\gamma}\right) / \mathcal{I}_{\omega \gamma}$}

By Theorem 3.1, the poset $\langle\mathbb{P}(\alpha), \subset\rangle$ is forcing equivalent to a forcing product of iterated reduced products of Boolean algebras of the form $P\left(\omega^{\gamma}\right) / \mathcal{I}_{\omega \gamma}$. In this section we consider such algebras and assume that $\gamma \geq \omega$ is a countable limit ordinal, $\left\langle\delta_{n}: n \in \omega\right\rangle$ a fixed increasing cofinal sequence in $\gamma \backslash\{0\}$ and $\mathbb{L}=\langle L,<\rangle$ $=\sum_{n \in \omega}\left\langle L_{n},<_{n}\right\rangle$, where $\left\langle L_{n},<_{n}\right\rangle \cong\left\langle\omega^{\delta_{n}}, \in\right\rangle$, for $n \in \omega$, and $L_{m} \cap L_{n}=\emptyset$, for $m \neq n$. For $A \subset L$ and $m \in \omega$ let $S_{A}^{m}=\left\{n \in \omega: \operatorname{type}\left(A \cap L_{n}\right) \geq \omega^{\delta_{m}}\right\}$ and $\operatorname{supp} A=\left\{n \in \omega: A \cap L_{n} \neq \emptyset\right\}$.

The ideal $\mathcal{I}_{\mathbb{L}}=\{A \subset L: \mathbb{L} \hookrightarrow A\}$ will be denoted by $\mathcal{I}$ and, if $G \subset P(\omega)$ is an ultrafilter, $\mathcal{I}_{G}=\{A \subset L: \exists I \in \mathcal{I} \operatorname{supp}(A \backslash I) \notin G\}$. $\Gamma$ (resp. $\left.\Gamma_{1}\right)$ will be the canonical name for an $\left\langle[\omega]^{\omega}, \subset^{*}\right\rangle$-generic (resp. $(P(\omega) / \text { Fin })^{+}$-generic) filter over the ground model $V$ and $q: P(\omega) \rightarrow P(\omega) /$ Fin the quotient mapping.

The aim of this section is to prove the following statement. It follows from Propositions 4.6 and 4.9, given at the end of the section. 
Theorem 4.1. For each countable limit ordinal $\gamma$ we have:

(a) The partial orders $\left\langle\mathbb{P}\left(\omega^{\gamma}\right), \subset\right\rangle$ and $\left(P\left(\omega^{\gamma}\right) / \mathcal{I}_{\omega \gamma}\right)^{+}$are forcing equivalent to the two-step iteration $(P(\omega) / \mathrm{Fin})^{+} *\left(P(L) / \check{\mathcal{I}}_{\check{q}^{-1}\left[\Gamma_{1}\right]}\right)^{+}$;

(b) $[\omega] \Vdash “\left(P(L) / \check{\mathcal{I}}_{\check{q}^{-1}\left[\Gamma_{1}\right]}\right)^{+}$is an $\omega_{1}$-closed, separative and atomless poset".

Fact 4.2. Let $f: \omega \rightarrow \omega$ be an increasing function. Then

(a) $\omega^{\delta_{f(0)}}+\omega^{\delta_{f(1)}}+\cdots+\omega^{\delta_{f(m)}}=\omega^{\delta_{f(m)}}$, for each $m \in \omega$;

(b) $\sum_{n \in \omega} \omega^{\delta_{f(n)}}=\omega^{\gamma}$;

(c) $\mathbb{L} \cong \omega^{\gamma}$.

Proof. We prove (a) by induction. Assuming that (a) is true for $m \in \omega$ we have $\omega^{\delta_{f(0)}}+\cdots+\omega^{\delta_{f(m+1)}}=\omega^{\delta_{f(m)}}+\omega^{\delta_{f(m+1)}}=\omega^{\delta_{f(m)}} \cdot 1+\omega^{\delta_{f(m)}}+\left(\delta_{f(m+1)}-\delta_{f(m)}\right)=$ $\omega^{\delta_{f(m)}}\left(1+\omega^{\delta_{f(m+1)}-\delta_{f(m)}}\right)=\omega^{\delta_{f(m)}} \omega^{\delta_{f(m+1)}-\delta_{f(m)}}=\omega^{\delta_{f(m+1)}}$.

(b) By (a) and basic properties of ordinal arithmetic we have $\sum_{n \in \omega} \omega^{\delta_{f(n)}}=$ $\sup \left\{\sum_{n \leq m} \omega^{\delta_{f(n)}}: m \in \omega\right\}=\sup \left\{\omega^{\delta_{f(m)}}: m \in \omega\right\}=\omega^{\gamma}$.

(c) By (b) we have $\mathbb{L} \cong \sum_{n \in \omega} \omega^{\delta_{n}}=\omega^{\gamma}$.

Lemma 4.3. For $A \subset L$ and $m \in \omega$ we have:

(a) $S_{A}^{m} \subset \operatorname{supp} A \backslash m$;

(b) $m_{1}<m_{2} \Rightarrow S_{A}^{m_{1}} \supset S_{A}^{m_{2}}$;

(c) $A \subset B \Rightarrow S_{A}^{m} \subset S_{B}^{m}$;

(d) $A \in P(L) \backslash \mathcal{I}$ iff $S_{A}^{m} \in[\omega]^{\omega}$, for each $m \in \omega$;

(e) $A \in \mathcal{I}$ iff $S_{A}^{m}=\emptyset$, for some $m \in \omega$;

(f) $|\operatorname{supp}(A)|<\omega \Rightarrow A \in \mathcal{I}$;

(g) $S_{\bigcup_{k<l}^{m} A_{k}}^{m}=\bigcup_{k<l} S_{A_{k}}^{m}$;

(h) $A \subset_{\mathcal{I}} B$ iff $S_{A \backslash B}^{m}=\emptyset$, for some $m \in \omega$.

Proof. (a), (b), (c) and (f) are evident and (h) follows from (e).

(d) By Fact 2.3, $A \in P(L) \backslash \mathcal{I}$ iff for each $m \in \omega$ we have: for each $n \in \omega$ there is finite $K \subset \omega \backslash n$ such that $L_{m} \cong \omega^{\delta_{m}} \hookrightarrow \bigcup_{i \in K} A \cap L_{j}$, but, by Fact 2.4. $\omega^{\delta_{m}}$ is an indivisible structure and, hence, this holds iff there is $k \geq n$ such that $\omega^{\delta_{m}} \hookrightarrow A \cap L_{k}$, that is, $k \in S_{A}^{m}$.

(e) By (c), if $S_{A}^{m}=\emptyset$ for some $m \in \omega$, then $A \in \mathcal{I}$. On the other hand, if $A \in \mathcal{I}$, then, by (c) again, there are $k, l \in \omega$ such that $S_{A}^{k} \subset l$ and, by (a) and (b), for $m \geq l, k$ we have $S_{A}^{m} \subset S_{A}^{k} \backslash m \subset S_{A}^{k} \backslash l=\emptyset$.

(g) If $n \in S_{\bigcup_{k<l}^{m} A_{k}}^{m}$, then $\omega^{\delta_{m}} \hookrightarrow \bigcup_{k<l} A_{k} \cap L_{n}$ and, since $\mathcal{I}_{\omega^{\delta_{m}}}=\left\{I \subset \omega^{\delta_{m}}\right.$ : $\left.\omega^{\delta_{m}} \hookrightarrow I\right\}$ is an ideal, there is $k<l$ such that $\omega^{\delta_{m}} \hookrightarrow A_{k} \cap L_{n}$, that is, $k \in S_{A_{k}}^{m}$. On the other hand, by (c) we have $S_{A_{k}}^{m} \subset S_{\bigcup_{k<l} A_{k}}^{m}$, for each $k<l$.

Lemma 4.4. If $G \subset P(\omega)$ is an ultrafilter, then

(a) $\mathcal{I}_{G}=\{A \subset L: \exists I \in \mathcal{I} \operatorname{supp}(A \backslash I) \notin G\}$ is an ideal and $\mathcal{I} \subset \mathcal{I}_{G}$;

(b) $\operatorname{sm}\left\langle P(L) \backslash \mathcal{I}_{G}, \subset_{\mathcal{I}}\right\rangle=\left\langle P(L) \backslash \mathcal{I}_{G}, \subset_{\mathcal{I}_{G}}\right\rangle$.

Proof. (a) If $A_{1}, A_{2} \in \mathcal{I}_{G}$ and $\operatorname{supp}\left(A_{1} \backslash I_{1}\right), \operatorname{supp}\left(A_{2} \backslash I_{2}\right) \notin G$, where $I_{1}, I_{2} \in \mathcal{I}$, then, since $\left(A_{1} \cup A_{2}\right) \backslash\left(I_{1} \cup I_{2}\right) \subset\left(A_{1} \backslash I_{1}\right) \cup\left(A_{2} \backslash I_{2}\right)$ and $\operatorname{supp}(X \cup Y)=\operatorname{supp}(X) \cup$ $\operatorname{supp}(Y)$, we have $\operatorname{supp}\left(\left(A_{1} \cup A_{2}\right) \backslash\left(I_{1} \cup I_{2}\right)\right) \subset \operatorname{supp}\left(A_{1} \backslash I_{1}\right) \cup \operatorname{supp}\left(A_{2} \backslash I_{2}\right) \notin G$ and, since $I_{1} \cup I_{2} \in \mathcal{I}$, we have $A_{1} \cup A_{2} \in \mathcal{I}_{G}$.

(b) Let $A \subset_{\mathcal{I}_{G}} B$ and $C \in P(L) \backslash \mathcal{I}_{G}$, where $C \subset_{\mathcal{I}} A$. Then, since $C=$ $(C \backslash A) \cup(C \cap A \backslash B) \cup(C \cap A \cap B), A \backslash B \in \mathcal{I}_{G}$ and $C \backslash A \in \mathcal{I} \subset \mathcal{I}_{G}$, we have $D=C \cap A \cap B \in P(L) \backslash \mathcal{I}_{G}$ and $D \subset_{\mathcal{I}} C, B$. Thus $A \subset_{\mathcal{I}}^{*} B$. Conversely, suppose 
that $A \subset_{\mathcal{I}}^{*} B$ and $A \backslash B \notin \mathcal{I}_{G}$. Then, for $C=A \backslash B$ there is $D \in P(L) \backslash \mathcal{I}_{G}$ such that $D \subset_{\mathcal{I}} A \backslash B$ and $D \subset_{\mathcal{I}} B$, which implies $D \in \mathcal{I}$. A contradiction.

We remind the reader that, if $\left\langle\mathbb{P} \leq_{\mathbb{P}}, 1_{\mathbb{P}}\right\rangle$ and $\left\langle\mathbb{Q} \leq_{\mathbb{Q}}, 1_{\mathbb{Q}}\right\rangle$ are pre-orders, then a mapping $f: \mathbb{P} \rightarrow \mathbb{Q}$ is a complete embedding, in notation $f: \mathbb{P} \hookrightarrow_{c} \mathbb{Q}$ iff

(ce1) $p_{1} \leq_{\mathbb{P}} p_{2} \Rightarrow f\left(p_{1}\right) \leq_{\mathbb{Q}} f\left(p_{2}\right)$,

(ce2) $p_{1} \perp_{\mathbb{P}} p_{2} \Leftrightarrow f\left(p_{1}\right) \perp_{\mathbb{Q}} f\left(p_{2}\right)$,

(ce3) $\forall q \in \mathbb{Q} \exists p \in \mathbb{P} \forall p^{\prime} \leq_{\mathbb{P}} p \quad f\left(p^{\prime}\right) \perp_{\mathbb{Q}} q$.

Then, for $q \in \mathbb{Q}$ the set $\operatorname{red}(q)=\left\{p \in \mathbb{P}: \forall p^{\prime} \leq_{\mathbb{P}} p f\left(p^{\prime}\right) \not \chi_{\mathbb{Q}} q\right\}$ is the set of reductions of $q$ to $\mathbb{P}$. The following fact is folklore (see [5]).

Fact 4.5. If $f: \mathbb{P} \hookrightarrow_{c} \mathbb{Q}$, then $\mathbb{Q}$ is forcing equivalent to the two-step iteration $\mathbb{P} *\left\langle\pi, \leq_{\pi}, \check{1_{\mathbb{Q}}}\right\rangle$, where $1_{\mathbb{P}} \Vdash_{\mathbb{P}} \pi \subset \check{\mathbb{Q}}$ and for each $p \in \mathbb{P}$ and $q, q_{1}, q_{2} \in \mathbb{Q}$

(a) $p \Vdash \check{q} \in \pi$ iff $p \in \operatorname{red}(q)$;

(b) $p \Vdash \check{q_{1}} \leq_{\pi} \check{q_{2}}$ iff $q_{1} \leq_{\mathbb{Q}} q_{2}$ and $p \in \operatorname{red}\left(q_{1}\right)$.

Proposition 4.6. The following pre-orders are forcing equivalent:
1. $\left\langle\mathbb{P}\left(\omega^{\gamma}\right), \subset\right\rangle$,
2. $\left(P\left(\omega^{\gamma}\right) / \mathcal{I}_{\omega \gamma}\right)^{+}$,
3. $\langle P(L) \backslash \mathcal{I}, \subset \mathcal{I}\rangle$,
4. $\left\langle[\omega]^{\omega}, \subset^{*}\right\rangle *\left\langle P(L) \backslash \check{\mathcal{I}}_{\Gamma}, \subset_{\check{\mathcal{I}}_{\Gamma}}\right\rangle$,
5. $(P(\omega) / \mathrm{Fin})^{+} *\left(P(L) / \check{\mathcal{I}}_{\check{q}^{-1}\left[\Gamma_{1}\right]}\right)^{+}$.

Proof. By Facts 2.5, 2.6(a) and (e) the posets 1 and 2 are forcing equivalent. By Facts 2.5] 2.1(b) and 2.6 (a), (d) the poset $\left\langle\mathbb{P}\left(\omega^{\gamma}\right), \subset\right\rangle=\left\langle P\left(\omega^{\gamma}\right) \backslash \mathcal{I}_{\omega \gamma}, \subset\right\rangle$ is isomorphic to the poset $\langle P(L) \backslash \mathcal{I}, \subset\rangle$, forcing equivalent to $\left\langle P(L) \backslash \mathcal{I}, \subset_{\mathcal{I}}\right\rangle$. The forcing equivalence of the posets 4 and 5 is evident - note that $G_{1}$ is a $(P(\omega) / \text { Fin })^{+}$-generic filter iff $G=q^{-1}\left[G_{1}\right]$ is an $\left\langle[\omega]^{\omega}, \subset^{*}\right\rangle$-generic filter over $V$ and that $\operatorname{sq}\left\langle P(L) \backslash \mathcal{I}_{G}, \subset_{\mathcal{I}_{G}}\right\rangle$ $=\left(P(L) / \mathcal{I}_{G}\right)^{+}$.

Thus the forcing equivalence of the posets 3 and 4 remains to be proved.

Claim 4.7. The mapping $f:\left\langle[\omega]^{\omega}, \subset^{*}\right\rangle \rightarrow\left\langle P(L) \backslash \mathcal{I}, \subset_{\mathcal{I}}\right\rangle$ defined by $f(S)=$ $\bigcup_{n \in S} L_{n}$ is a complete embedding. In addition, $\mathfrak{t}\left(\left\langle P(L) \backslash \mathcal{I}, \subset_{\mathcal{I}}\right\rangle\right) \leq \mathfrak{t}$.

Proof. By Fact 4.2 (b) and (c), for $S \in[\omega]^{\omega}$ we have $f(S) \cong \sum_{n \in S} \omega^{\delta_{n}}=\omega^{\gamma} \cong \mathbb{L}$, thus $f(S) \in P(L) \backslash \mathcal{I}$. Let $S, T \in[\omega]^{\omega}$.

(ce1) If $S \subset^{*} T$, then $|\operatorname{supp}(f(S) \backslash f(T))|=\left|\operatorname{supp}\left(\bigcup_{n \in S \backslash T} L_{n}\right)\right|=|S \backslash T|<\omega$ and, by Lemma 4.3(f), $f(S) \backslash f(T) \in \mathcal{I}$, that is, $f(S) \subset_{\mathcal{I}} f(T)$.

(ce2) If $S \perp T$, then $|\operatorname{supp}(f(S) \cap f(T))|=\left|\operatorname{supp}\left(\bigcup_{n \in S \cap T} L_{n}\right)\right|=|S \cap T|<\omega$ and, by Lemma 4.3. (f), $f(S) \cap f(T) \in \mathcal{I}$, that is, $f(S) \perp_{\mathcal{I}} f(T)$. If $S \not \perp T$, then $S \cap T \in[\omega]^{\omega}$ and $f(S) \cap f(T)=\bigcup_{n \in S \cap T} L_{n}=f(S \cap T) \in P(L) \backslash \mathcal{I}$ and, hence, $f(S) \not \not_{\mathcal{I}} f(T)$.

(ce3) First we show that for $S \in[\omega]^{\omega}$ and $A \in P(L) \backslash \mathcal{I}$ we have

$$
S \in \operatorname{red}(A) \text { iff } S \subset^{*} S_{A}^{m} \text {, for each } m \in \omega \text {. }
$$

Suppose that $S \in \operatorname{red}(A)$ and that $T=S \backslash S_{A}^{m} \in[\omega]^{\omega}$, for some $m \in \omega$. Then there is $B \in P(L) \backslash \mathcal{I}$ such that $B \subset_{\mathcal{I}} f(T), A$. Now we use Lemma 4.3, By (h), there are $m_{1}, m_{2} \in \omega$ such that $S_{B \backslash f(T)}^{m_{1}}=S_{B \backslash A}^{m_{2}}=\emptyset$. By (b), for $m^{*}=\max \left\{m, m_{1}, m_{2}\right\}$ 
we have $S_{B \backslash f(T)}^{m^{*}}=S_{B \backslash A}^{m^{*}}=\emptyset$ and, by $(\mathrm{g}), S_{B}^{m^{*}}=S_{(B \cap A \cap f(T)) \cup(B \backslash f(T)) \cup(B \backslash A)}^{m^{*}}=$ $S_{B \cap A \cap f(T)}^{m^{*}} \cup S_{B \backslash f(T)}^{m^{*}} \cup S_{B \backslash A}^{m^{*}}=S_{B \cap A \cap f(T)}^{m^{*}}$. But, by (a), (b) and (c), $S_{B \cap A \cap f(T)}^{m^{*}} \subset$ $S_{f(T)}^{m^{*}} \cap S_{A}^{m^{*}} \subset T \cap S_{A}^{m}=\emptyset$, that is, $S_{B}^{m^{*}}=\emptyset$, which, by (e), implies $B \in \mathcal{I}$. A contradiction.

Let $S \subset^{*} S_{A}^{m}$, for each $m \in \omega$, and let $[\omega]^{\omega} \ni T \subset^{*} S$. In order to find a set $B \in P(L) \backslash \mathcal{I}$ such that $B \subset_{\mathcal{I}} f(T), A$ by recursion we construct a sequence $\left\langle n_{k}: k \in \omega\right\rangle$ such that for each $k \in \omega$ we have: (i) $n_{k} \in T$, (ii) $n_{k}<n_{k+1}$ and (iii) type $\left(A \cap L_{n_{k}}\right) \geq \omega^{\delta_{k}}$. If a sequence $\left\langle n_{0}, \ldots, n_{k}\right\rangle$ satisfies (i)-(iii), then for $n_{k+1}=\min \left(T \cap S_{A}^{k+1} \backslash\left(n_{k}+1\right)\right)$ we have $n_{k}<n_{k+1}$ and type $\left(A \cap L_{n_{k+1}}\right) \geq \omega^{\delta_{k+1}}$. Thus, the recursion works. Now $B=\bigcup_{k \in \omega} A \cap L_{n_{k}} \subset A$, by (i) we have $B \subset f(T)$ and, by (iii), for each $k \in \omega$ we have $S_{B}^{k} \supset\left\{n_{k}, n_{k+1}, \ldots\right\}$. Thus, by Lemma 4.3(d) we have $B \in P(L) \backslash \mathcal{I}$ and (4.1) is proved.

Now we check (ce3). If $A \in P(L) \backslash \mathcal{I}$, then, by Lemma $4.3\left\{S_{A}^{m}: m \in \omega\right\}$ is a subfamily of $[\omega]^{\omega}$ having the strong finite intersection property and, hence, it has a pseudointersection $S \in[\omega]^{\omega}$. By (4.1), $S$ is a reduction of $A$ to $[\omega]^{\omega}$.

If $\left\langle T_{\alpha}: \alpha<\mathfrak{t}\right\rangle$ is a tower in $\left\langle[\omega]^{\omega}, \subset^{*}\right\rangle$, then, by (ce1), $\left\langle f\left(T_{\alpha}\right): \alpha<\mathfrak{t}\right\rangle$ is a $\subset_{\mathcal{I}}$-decreasing sequence in $P(L) \backslash \mathcal{I}$. Suppose that $A \in P(L) \backslash \mathcal{I}$ and that for each $\alpha<\mathfrak{t}$ we have $A \subset_{\mathcal{I}} f\left(T_{\alpha}\right)$, which, by Lemma 4.3(h), gives $m_{\alpha} \in \omega$ such that $S_{A \backslash f\left(T_{\alpha}\right)}^{m_{\alpha}}=\emptyset$ and, by Lemma $4.3(\mathrm{~g}), S_{A}^{m_{\alpha}}=S_{A \cap f\left(T_{\alpha}\right)}^{m_{\alpha}} \subset T_{\alpha}$. Let $S \in \operatorname{red}(A)$. Then, by (4.1), $S \subset^{*} S_{A}^{m_{\alpha}} \subset T_{\alpha}$, for each $\alpha<\mathfrak{t}$. A contradiction.

By the previous claim, Fact 4.5 and (4.1), the pre-order $\left\langle P(L) \backslash \mathcal{I}, \subset_{\mathcal{I}}\right\rangle$ is forcing equivalent to the iteration $\left\langle[\omega]^{\omega}, \subset^{*}\right\rangle *\left\langle\pi, \leq_{\pi}, \check{L}\right\rangle$, where $\omega \Vdash \pi \subset(P(L) \backslash \mathcal{I})^{-}$and for each $S \in[\omega]^{\omega}$ and $A, B \in P(L) \backslash \mathcal{I}$ we have

$$
\begin{aligned}
S \Vdash \check{A} \in \pi & \Leftrightarrow \quad \forall m \in \omega S \subset^{*} S_{A}^{m} ; \\
S \Vdash \check{A} \leq_{\pi} \check{B} & \Leftrightarrow \quad S \Vdash \check{A} \in \pi \wedge A \subset_{\mathcal{I}} B .
\end{aligned}
$$

Claim 4.8. (a) $\omega \Vdash$ " $\check{\mathcal{I}}$ and $\check{\mathcal{I}}_{\Gamma}$ are ideals in $P(\check{L})=P(L)^{\prime \prime}$;

(b) $\omega \Vdash \pi=P(L) \backslash \check{\mathcal{I}}_{\Gamma}$;

(c) $\omega \Vdash \leq_{\pi}=C_{\check{\mathcal{I}}} \cap(\pi \times \pi)$.

Proof. Let $G$ be an $\left\langle[\omega]^{\omega}, \subset^{*}\right\rangle$-generic filter over $V$.

(a) Since the forcing $\left\langle[\omega]^{\omega}, \subset^{*}\right\rangle$ is $\omega$-distributive, in $V[G]$ we have $P^{V[G]}(L)=$ $P^{V}(L)$ and, for the same reason, $\mathcal{I}$ remains to be an ideal in $P^{V[G]}(L)$. By Lemma 4.4(a), the set $\mathcal{I}_{G}=\{A \subset L: \exists I \in \mathcal{I} \operatorname{supp}(A \backslash I) \notin G\}$ is an ideal in $P^{V[G]}(L)$.

(b) We show that $\pi_{G}=\{A \in P(L) \backslash \mathcal{I}: \forall I \in \mathcal{I} \operatorname{supp}(A \backslash I) \in G\}$. Let $A \in P(L) \backslash \mathcal{I}$. If $A \in \pi_{G}$, then there is $S \in G$ such that $S \Vdash \check{A} \in \pi$. For $I \in \mathcal{I}$ we have $A \cap I \in \mathcal{I}$ and, by Lemma 4.3(e), there is $m^{*} \in \omega$ such that $S_{A \cap I}^{m^{*}}=\emptyset$. Thus, by (4.2) and Lemma 4.3(g) we have $S \subset^{*} S_{A}^{m^{*}}=S_{A \cap I}^{m^{*}} \cup S_{A \backslash I}^{m^{*}}=S_{A \backslash I}^{m^{*}} \subset \operatorname{supp}(A \backslash I)$, which implies that $\operatorname{supp}(A \backslash I) \in G$. So $A \in P(L) \backslash \mathcal{I}_{G}$.

If $A \notin \pi_{G}$, then there is $S \in G$ such that $S \Vdash \neg \check{A} \in \pi$. Suppose that $\left|S \cap S_{A}^{m}\right|=\omega$, for each $m \in \omega$. Then, by Lemma 4.3(b), $S \cap S_{A}^{m}, m \in \omega$, would be a decreasing sequence in $[\omega]^{\omega}$ and, hence, there would be $T \in[\omega]^{\omega}$ such that $T \subset^{*} S \cap S_{A}^{m}$, for each $m \in \omega$, which, by (4.2), implies $T \Vdash \check{A} \in \pi$. But this is impossible since $T \subset^{*} S$ and $S \Vdash \neg \check{A} \in \pi$. Thus $\left|S \cap S_{A}^{m^{*}}\right|<\omega$, for some $m^{*} \in \omega$. Let $I=\bigcup_{n \in S} A \cap L_{n}$. By Lemma 4.3(c) we have $S_{I}^{m^{*}} \subset S \cap S_{A}^{m^{*}}$ and, hence, $\left|S_{I}^{m^{*}}\right|<\omega$, which, by Lemma 4.3 (d), implies $I \in \mathcal{I}$. Since $\operatorname{supp}(A \backslash I) \cap S=\emptyset$ and $S \in G$, we have $\operatorname{supp}(A \backslash I) \notin G$. So $A \notin P(L) \backslash \mathcal{I}_{G}$. 
(c) We show that $\left(\leq_{\pi}\right)_{G}=\subset_{\mathcal{I}} \cap\left(P(L) \backslash \mathcal{I}_{G}\right)^{2}$. Let $A, B \in \pi_{G}$ and let $S \in G$ where $S \Vdash \check{A}, \check{B} \in \pi$. If $A\left(\leq_{\pi}\right)_{G} B$, then there is $T \in G$ such that $T \Vdash \check{A} \leq_{\pi} \check{B}$, which, by (4.3), implies $A \subset_{\mathcal{I}} B$. If $A \subset_{\mathcal{I}} B$, then, since $S \Vdash \check{A} \in \pi$, by (4.3) we have $S \Vdash \check{A} \leq_{\pi} \check{B}$ and, hence, $A\left(\leq_{\pi}\right)_{G} B$.

Thus, the pre-order $\left\langle P(L) \backslash \mathcal{I}, \subset_{\mathcal{I}}\right\rangle$ is forcing equivalent to the two-step iteration $\left\langle[\omega]^{\omega}, \subset^{*}\right\rangle *\left\langle\check{(L)} \backslash \check{\mathcal{I}}_{\Gamma}, \check{C_{\mathcal{I}}}\right\rangle$ and, by Lemma 4.4 (b) applied in $V[G]$, to the iteration $\left\langle[\omega]^{\omega}, \subset^{*}\right\rangle *\left\langle P(L) \backslash \check{\mathcal{I}}_{\Gamma}, \subset_{\mathcal{I}_{\Gamma}}\right\rangle$.

Proposition 4.9. According to the notation of Proposition 4.6 we have

(a) $\omega \Vdash "\left\langle P(L) \backslash \check{\mathcal{I}}_{\Gamma}, \subset_{\check{\mathcal{I}}_{\Gamma}}\right\rangle$ is a separative, $\omega_{1}$-closed and atomless pre-order".

(b) $[\omega] \Vdash "\left(P(L) / \check{\mathcal{I}}_{\breve{q}^{-1}\left[\Gamma_{1}\right]}\right)+$ is a separative, $\omega_{1}$-closed and atomless poset."

Proof. (a) The separativity follows from Fact 2.6(d) and we prove $\omega_{1}$-closure. We easily show that for $S \in[\omega]^{\omega}$ and $A, B \in P(L)$ satisfying $S \Vdash \check{A}, \check{B} \in \pi$ we have:

$$
\left.\left.S \Vdash \check{A} \subset_{\check{\mathcal{I}}_{\Gamma}} \check{B} \Leftrightarrow \forall T \subset^{*} S \quad \exists I \in \mathcal{I} \mid T \backslash \operatorname{supp}(A \backslash B) \backslash I\right)\right) \mid=\omega .
$$

Since the forcing $\left\langle[\omega]^{\omega}, \subset^{*}\right\rangle$ is $\omega$-distributive we have $\omega \Vdash P(L)^{\check{\omega}}=\left(\left(P(L)^{\omega}\right)^{V}\right)^{-}$ and, clearly, $\omega \Vdash \pi \subset P(L)$. So, assuming that $\left\langle A_{n}: n \in \omega\right\rangle \in P(L)^{\omega}, S \in[\omega]^{\omega}$ and $S \Vdash \forall n \in \check{\omega}\left(\check{A_{n}} \in \pi \wedge \forall m \geq n \check{A_{m}} \subset_{\check{\mathcal{I}}_{\Gamma}} \check{A_{n}}\right)$, that is, by (4.2) and (4.4),

$$
\begin{gathered}
\forall m, n \in \omega S \subset^{*} S_{A_{n}}^{m}, \\
\forall R \subset^{*} S \exists I \in \mathcal{I}\left|R \backslash \operatorname{supp}\left(\left(A_{n+1} \backslash A_{n}\right) \backslash I\right)\right|=\omega,
\end{gathered}
$$

it is sufficient to find $T \in[\omega]^{\omega}$ and $A \in P(L)$ such that $T \subset^{*} S, T \Vdash \check{A} \in \pi$ and $T \Vdash \check{A} \subset_{\check{\mathcal{I}}_{\Gamma}} \check{A_{n}}$, for all $n \in \omega$.

Claim 4.10. For $r \in \omega$, let $S_{r}=S \cap \bigcap_{m, n \leq r} S_{A_{n}}^{m}$ and $B_{r}=A_{r} \cap \bigcup_{k \in S_{r}} L_{k}$. Then

(a) $B_{r} \in P(L) \backslash \mathcal{I}$

(b) $B_{r+1} \subset_{\mathcal{I}} B_{r}$.

Proof. (a) If $m \in \omega$, then $k \in S_{B_{r}}^{m}$ iff $k \in S_{r}$ and $\omega^{\delta_{m}} \hookrightarrow B_{r} \cap L_{k}=A_{r} \cap L_{k}$; thus $S_{B_{r}}^{m}=S_{r} \cap S_{A_{r}}^{m}$ and, by (4.5), $\left|S_{B_{r}}^{m}\right|=\omega$. Now, by Lemma 4.3(d), $B_{r} \in P(L) \backslash \mathcal{I}$.

(b) Suppose that $B_{r+1} \not \subset_{\mathcal{I}} B_{r}$. Then, since $S_{r+1} \subset S_{r}$, we would have $C=$ $B_{r+1} \backslash B_{r}=\left(A_{r+1} \backslash A_{r}\right) \cap \bigcup_{k \in S_{r+1}} L_{k} \in P(L) \backslash \mathcal{I}$ and, by Lemma 4.3(b) and (d), there would be $R \in[\omega]^{\omega}$ such that $R \subset^{*} S_{C}^{m}$, for all $m \in \omega$. Since $S_{C}^{m} \subset$ $\operatorname{supp}(C) \subset S_{r+1} \subset S$ we would have $R \subset^{*} S$ and, by (4.6), there would be $I \in \mathcal{I}$ such that $\left.R \not C^{*} \operatorname{supp}\left(\left(A_{r+1} \backslash A_{r}\right) \backslash I\right)\right)$. Since $\left(A_{r+1} \backslash A_{r}\right) \cap I \in \mathcal{I}$, by Lemma 4.3(e) there is $m^{*} \in \omega$ such that $S_{\left(A_{r+1} \backslash A_{r}\right) \cap I}^{m^{*}}=\emptyset$ and, by Lemma 4.3 $(\mathrm{g}), S_{A_{r+1} \backslash A_{r}}^{m^{*}}=$ $S_{\left(A_{r+1} \backslash A_{r}\right) \backslash I}^{m^{*}} \subset \operatorname{supp}\left(\left(A_{r+1} \backslash A_{r}\right) \backslash I\right)$. But, by Lemma 4.3)(c), $R \subset{ }^{*} S_{C}^{m^{*}} \subset S_{A_{r+1} \backslash A_{r}}^{m^{*}}$, thus $R \subset^{*} \operatorname{supp}\left(\left(A_{r+1} \backslash A_{r}\right) \backslash I\right)$. A contradiction.

By Theorem 1.1 the pre-order $\left\langle P(L) \backslash \mathcal{I}, \subset_{\mathcal{I}}\right\rangle$ is $\omega_{1}$-closed so, by Claim 4.10 , there is $A \in P(L) \backslash \mathcal{I}$ such that

$$
\forall n \in \omega A \subset_{\mathcal{I}} B_{n} \subset A_{n} .
$$

By Lemma 4.3(b) and (d) there is $T \in[\omega]^{\omega}$ such that 
By (4.7) we have $A \backslash B_{n} \in \mathcal{I}$, by Lemma 4.3(e) there is $m^{*} \in \omega$ such that $S_{A \backslash B_{n}}^{m^{*}}=$ $\emptyset$ and, by Lemma 4.3(g) we have $S_{A}^{m^{*}}=S_{A \cap B_{n}}^{m^{*}} \cup S_{A \backslash B_{n}}^{m^{*}}=S_{A \cap B_{n}}^{m^{*}} \subset S_{B_{n}}^{m^{*}} \subset$ $\operatorname{supp}\left(B_{n}\right) \subset S_{n} \subset S$. By (4.8) we have $T \subset^{*} S_{A}^{m^{*}}$ and, hence, $T \subset^{*} S$. By (4.8) and (4.2) we have $T \Vdash \check{A} \in \pi$. By (4.7), for each $n \in \omega$ we have $A \subset_{\mathcal{I}} A_{n}$ and, hence, $T \Vdash \check{A} \subset_{\check{\mathcal{I}}_{\Gamma}} \check{A}_{n}$.

Taking an $\left\langle[\omega]^{\omega}, \subset^{*}\right\rangle$-generic filter $G$ we prove that the pre-order $\left\langle\pi_{G}, \subset_{\check{\mathcal{I}}_{G}}\right\rangle$ is atomless. If $A \in \pi_{G}$, then, by (4.2), there is $S \in G$ such that $S \subset^{*} S_{A}^{m}$, for each $m \in \omega$. By Lemma 4.3(b) we have $S_{A}^{0} \supset S_{A}^{1} \supset \ldots$ and, clearly, $\bigcap_{m \in \omega} S_{A}^{m}=\emptyset$. W.l.o.g. suppose that $S \subset S_{A}^{0}$. Then $S=\bigcup_{m \in \omega} S \cap\left(S_{A}^{m} \backslash S_{A}^{m+1}\right)$ and, for $n \in$ $S \cap\left(S_{A}^{m} \backslash S_{A}^{m+1}\right)$ there is $\varphi_{n}: \omega^{\delta_{m}} \hookrightarrow A \cap L_{n}$. Let $\varphi_{n}\left[\omega^{\delta_{m}}\right]=B_{n} \dot{\cup} C_{n}$, where $B_{n}, C_{n} \cong \omega^{\delta_{m}}$ and let $B=\bigcup_{m \in S} B_{n}$ and $C=\bigcup_{m \in S} C_{n}$. Then $S_{B}^{m}=S \cap S_{A}^{m}$ and, hence, $S \subset^{*} S_{B}^{m}$, for all $m \in \omega$, which implies $B \in \pi_{G}$ and, similarly, $C \in \pi_{G}$. Since $B, C \subset A$ we have $B, C \subset_{\mathcal{I}_{G}} A$ and $B \cap C=\emptyset$ implies that $B$ and $C$ are $\subset_{\check{I}_{G}}$-incompatible.

The proof of (b) is similar to the proof of (a). Note that $\left\langle P(L) \backslash \mathcal{I}_{G}, \subset \mathcal{I}_{G}\right\rangle$ is $\omega_{1}$-closed (atomless) iff $\left(P(L) / \mathcal{I}_{G}\right)^{+}$is $\omega_{1}$-closed (atomless).

\section{Forcing With $\langle\mathbb{P}(\alpha), \subset\rangle$}

If $\mathbb{P}, \mathbb{Q}$ and $\mathbb{R}$ are pre-orders, then, clearly, $\mathbb{P} \times \mathbb{Q} \cong \mathbb{Q} \times \mathbb{P}$ and $(\mathbb{P} \times \mathbb{Q}) \times \mathbb{R} \cong$ $\mathbb{P} \times(\mathbb{Q} \times \mathbb{R})$, that is, concerning the forcing equivalence of pre-orders, direct product is a commutative and associative operation. The following lemma generalizes the associativity law.

Lemma 5.1. Let $\mathbb{P}$ and $\mathbb{Q}$ be pre-orders and $\left\langle\pi, \leq_{\pi}, 1_{\pi}\right\rangle$ a $\mathbb{P}$-name for a pre-order. Then there is a $\mathbb{P}$-name for a pre-order $\left\langle\pi_{1}, \leq_{\pi_{1}}, 1_{\pi_{1}}\right\rangle$ such that

(a) $(\mathbb{P} * \pi) \times \mathbb{Q} \cong \mathbb{P} * \pi_{1}$.

(b) If $\mathbb{P}$ is $\omega$-distributive, $1_{\mathbb{P}} \Vdash_{\mathbb{P}}$ " $\pi$ is $\omega_{1}$-closed" and $\mathbb{Q}$ is $\omega_{1}$-closed, then $1_{\mathbb{P}} \vdash_{\mathbb{P}}$ " $\pi_{1}$ is $\omega_{1}$-closed".

(c) If $1_{\mathbb{P}} \Vdash_{\mathbb{P}}$ " $\pi$ is separative" and $\mathbb{Q}$ is separative, then $1_{\mathbb{P}} \Vdash_{\mathbb{P}}$ " $\pi_{1}$ is separative".

(d) If $1_{\mathbb{P}} \Vdash_{\mathbb{P}}$ " $\pi$ is atomless" or $\mathbb{Q}$ is atomless, then $1_{\mathbb{P}} \Vdash_{\mathbb{P}}$ " $\pi_{1}$ is atomless".

Proof. It is easy to show that the triple $\left\langle\pi_{1}, \leq_{\pi_{1}}, 1_{\pi_{1}}\right\rangle$ works, where

$$
\begin{aligned}
\pi_{1} & \left.=\left\{\langle\langle\tau, q\rangle\rangle^{\sim}, p\right\rangle: p \in \mathbb{P} \wedge \tau \in \operatorname{dom} \pi \wedge q \in \mathbb{Q} \wedge p \Vdash_{\mathbb{P}} \tau \in \pi\right\}, \\
\leq_{\pi_{1}} & =\left\{\left\langle\left\langle\left\langle\tau_{0}, q_{0}\right\rangle,\left\langle\tau_{1}, q_{1}\right\rangle\right\rangle^{\sim}, p\right\rangle: p \Vdash_{\mathbb{P}} \tau_{0}, \tau_{1} \in \pi \wedge \tau_{0} \leq_{\pi} \tau_{1} \wedge q_{0} \leq_{\mathbb{Q}} q_{1}\right\}, \\
1_{\pi_{1}} & =\left\langle 1_{\pi}, 1_{\mathbb{Q}}\right\rangle .
\end{aligned}
$$

Fact 5.2. Let $\mathbb{B}$ be a non-trivial Boolean algebra, $\mathcal{U} \subset P(\omega)$ a non-principal ultrafilter and $\mathbb{B}^{\omega} / \mathcal{U}$ the corresponding ultrapower. Then

(a) The poset $\left(\mathbb{B}^{\omega} / \mathcal{U}\right)^{+}$is $\omega_{1}$-closed and separative (folklore).

(b) If the algebra $\mathbb{B}$ is atomless, then $\left(\mathbb{B}^{\omega} / \mathcal{U}\right)^{+}$is an atomless poset (folklore).

(c) (See [3].) The poset $(\operatorname{rp}(\mathbb{B}))^{+}$is forcing equivalent to the two-step iteration $(P(\omega) / \text { Fin })^{+} *\left(\mathbb{B}^{\omega} / \Gamma_{1}\right)^{+}$.

Theorem 5.3. For each countable ordinal $\alpha \geq \omega+\omega$ the partial order $\langle\mathbb{P}(\alpha), \subset\rangle$ is forcing equivalent to a two-step iteration of the form $(P(\omega) / \text { Fin })^{+} * \pi$, where $[\omega] \Vdash$ " $\pi$ is an $\omega_{1}$-closed, separative atomless forcing". 
Proof. Using the notation of Theorem 3.1 for $\alpha=\omega^{\gamma_{n}+r_{n}} s_{n}+\cdots+\omega^{\gamma_{0}+r_{0}} s_{0}+k$ we have $\operatorname{sq}\langle\mathbb{P}(\alpha), \subset\rangle \cong \prod_{i=0}^{n}\left(\left(\operatorname{rp}^{r_{i}}\left(P\left(\omega^{\gamma_{i}}\right) / \mathcal{I}_{\omega \gamma_{i}}\right)\right)^{+}\right)^{s_{i}}$.

If $r_{i}=0$, for all $i \leq n$, then $\alpha=\omega^{\gamma_{n}} s_{n}+\cdots+\omega^{\gamma_{0}} s_{0}+k$, where $\gamma_{n} \in$ Lim or $\gamma_{n}=1$, and $\operatorname{sq}\langle\mathbb{P}(\alpha), \subset\rangle \cong \prod_{i=0}^{n}\left(\left(P\left(\omega^{\gamma_{i}}\right) / \mathcal{I}_{\omega \gamma_{i}}\right)^{+}\right)^{s_{i}}$. So, if $\gamma_{n} \geq \omega$, then, by the associativity of direct products, $\mathrm{sq}\langle\mathbb{P}(\alpha), \subset\rangle \cong\left(P\left(\omega^{\gamma_{n}}\right) / \mathcal{I}_{\omega \gamma_{n}}\right)^{+} * \mathbb{Q}$, where $\mathbb{Q}$ is an $\omega_{1}$-closed, separative and atomless poset (see Theorem 1.1 and Facts 2.5 and 2.7(a)). Thus, by Theorem 4.1, the poset $\mathrm{sq}\langle\mathbb{P}(\alpha), \subset\rangle$ is forcing equivalent to the product $\mathbb{R}=\left((P(\omega) / \text { Fin })^{+} * \pi\right) \times \mathbb{Q}$, where $[\omega] \Vdash " \pi$ is an $\omega_{1}$-closed, separative atomless forcing" and, by Lemma $5.1, \mathbb{R}$ forcing equivalent to an iteration $(P(\omega) / \mathrm{Fin})^{+} * \pi_{1}$, where $[\omega] \Vdash$ " $\pi_{1}$ is an $\omega_{1}$-closed, separative atomless forcing". If $\gamma_{n}=1$, then $\alpha=\omega \cdot s_{n}$ and, by the assumption, $s_{n} \geq 2$. Thus $\operatorname{sq}\langle\mathbb{P}(\alpha), \subset\rangle \cong(P(\omega) / \text { Fin })^{+} \times$ $\left((P(\omega) / \text { Fin })^{+}\right)^{s_{n}-1}=(P(\omega) / \text { Fin })^{+} \times \pi$, where $\pi=\left(\left((P(\omega) / \text { Fin })^{+}\right)^{s_{n}-1}\right)^{\swarrow}$.

If $r_{i_{0}}>0$, for some $i_{0} \leq n$, then, by the associativity and commutativity of direct products, $\mathrm{sq}\langle\mathbb{P}(\alpha), \subset\rangle \cong\left(\operatorname{rp}\left(\mathrm{rp}^{r_{i_{0}}-1}\left(P\left(\omega^{\gamma_{i_{0}}}\right) / \mathcal{I}_{\omega^{\gamma_{i}}}\right)\right)\right)^{+} \times \mathbb{Q}$, where $\mathbb{Q}$ is an $\omega_{1}$-closed, separative and atomless poset (see Theorem 1.1, Lemma 3.2 and Fact 2.7(a)). If $\mathbb{B}=\operatorname{rp}^{r_{i_{0}}-1}\left(P\left(\omega^{\gamma_{i_{0}}}\right) / \mathcal{I}_{\omega^{\gamma_{i_{0}}}}\right)$, then, by Fact $5.2(\mathrm{c}), \mathrm{sq}\langle\mathbb{P}(\alpha), \subset\rangle$ is forcing equivalent to the product $\left((P(\omega) / \text { Fin })^{+} * \pi\right) \times \mathbb{Q}$, where $[\omega] \Vdash \pi=\left(\widetilde{\mathbb{B}^{\omega}} / \Gamma_{1}\right)^{+}$, and, by Fact 5.2 (a) and (b) applied in extensions by $(P(\omega) / \text { Fin })^{+},[\omega] \Vdash$ " $\pi$ is an $\omega_{1}$-closed, separative atomless forcing". By Lemma [5.1, $\mathrm{sq}\langle\mathbb{P}(\alpha), \subset\rangle$ is forcing equivalent to an iteration $(P(\omega) / \text { Fin })^{+} * \pi_{1}$, where $[\omega] \Vdash$ " $\pi_{1}$ is an $\omega_{1}$-closed, separative atomless forcing".

Theorem 5.4. If $\mathfrak{h}=\omega_{1}$, then for each countable ordinal $\alpha \geq \omega$ the partial order $\langle\mathbb{P}(\alpha), \subset\rangle$ is forcing equivalent to $(P(\omega) / \text { Fin })^{+}$.

Proof. If $\alpha<\omega+\omega$, then, by Theorem 3.1, sq $\langle\mathbb{P}(\alpha), \subset\rangle \cong(P(\omega) / \text { Fin })^{+}$.

Otherwise, by Theorem 5.3, $\langle\mathbb{P}(\alpha), \subset\rangle$ is forcing equivalent to a two-step iteration $(P(\omega) / \text { Fin })^{+} * \pi$, where $[\omega] \Vdash$ " $\pi$ is an $\omega_{1}$-closed, separative atomless forcing". Now, $V \models \mathfrak{h}=\omega_{1}$ implies that $\mathrm{CH}$ holds in each generic extension $V_{(P(\omega) / \text { Fin })^{+}}[G]$ and, by Fact 2.7(b) applied in $V_{(P(\omega) / \text { Fin })^{+}}[G]$, the pre-order $\pi_{G}$ is forcing equivalent to $\left((P(\omega) / \text { Fin })^{+}\right)^{V[G]}$. But, since forcing by $(P(\omega) / \text { Fin })^{+}$does not produce reals, $\left((P(\omega) / \text { Fin })^{+}\right)^{V[G]}=\left((P(\omega) / \text { Fin })^{+}\right)^{V}$ and, hence, $\langle\mathbb{P}(\alpha), \subset\rangle$ is forcing equivalent to $(P(\omega) / \text { Fin })^{+} \times(P(\omega) / \text { Fin })^{+}$. Now, in $V$ we have $\mathfrak{c}^{<\omega_{1}}=\mathfrak{c}$ and the posets $(P(\omega) / \text { Fin })^{+}$and $(P(\omega) / \text { Fin })^{+} \times(P(\omega) / \text { Fin })^{+}$are $\omega_{1}$-closed of size $\mathfrak{c}$. In addition, $\mathfrak{h}=\omega_{1}$ implies that they collapse $\mathfrak{c}$ to $\omega_{1}$ and, by Fact 2.7(c) they are forcing equivalent (to $\left.\operatorname{Coll}\left(\omega_{1}, \mathfrak{c}\right)\right)$.

Example 5.5. If $\mathfrak{h}_{n}$ denotes the distributivity number of the poset $\left((P(\omega) / \mathrm{Fin})^{+}\right)^{n}$ then, clearly, $\mathfrak{h} \geq \mathfrak{h}_{2} \geq \mathfrak{h}_{3} \geq \cdots \geq \omega_{1}$ and, by Corollary $3.8, \mathfrak{h}(\operatorname{sq}\langle\mathbb{P}(\omega n), \subset\rangle)=\mathfrak{h}_{n}$. By a result of Shelah and Spinas 12 , for each $n \in \mathbb{N}$ there is a model of ZFC in which $\mathfrak{h}_{n+1}<\mathfrak{h}_{n}$ and, hence, the posets $\langle\mathbb{P}(\omega n), \subset\rangle$ and $\langle\mathbb{P}(\omega(n+1)), \subset\rangle$ are not forcing equivalent.

\section{Forcing With QUOTIENTS OVER ORDINAL IDEALS}

The ideals $\mathcal{I}_{\omega^{\delta}}=\left\{I \subset \omega^{\delta}: \omega^{\delta} \hookrightarrow \neg\right.$, where $0<\delta<\omega_{1}$, are called ordinal or indecomposable ideals. If $\delta=\gamma+r$, where $\gamma \in \operatorname{Lim} \cup\{1\}$ and $r \in \omega$, then, by Facts 2.5, 2.6 and Theorem 3.1, we have

$$
\operatorname{sq}\left\langle\mathbb{P}\left(\omega^{\delta}\right), \subset\right\rangle=\left(P\left(\omega^{\gamma+r}\right) / \mathcal{I}_{\omega \gamma+r}\right)^{+} \cong\left(\operatorname{rp}^{r}\left(P\left(\omega^{\gamma}\right) / \mathcal{I}_{\omega \gamma}\right)\right)^{+} .
$$


Let $\mathfrak{h}_{\omega^{\delta}}=\mathfrak{h}\left(\left(P\left(\omega^{\delta}\right) / \mathcal{I}_{\omega^{\delta}}\right)^{+}\right)$and $\mathfrak{t}_{\omega^{\delta}}=\mathfrak{t}\left(\left(P\left(\omega^{\delta}\right) / \mathcal{I}_{\omega^{\delta}}\right)^{+}\right)$. Then we have

Theorem 6.1. For each $\gamma \in \operatorname{Lim} \cup\{1\}$ we have

(a) $\mathfrak{h} \geq \mathfrak{h}_{\omega \gamma} \geq \mathfrak{h}_{\omega \gamma+1} \geq \cdots \geq \mathfrak{h}_{\omega \gamma+r} \geq \cdots \geq \omega_{1}$ and, hence, there is $r_{0} \in \omega$ such that $\mathfrak{h}_{\omega \gamma+r}=\mathfrak{h}_{\omega \gamma+r_{0}}$, for each $r \geq r_{0}$;

(b) $\mathfrak{t} \geq \mathfrak{t}_{\omega \gamma} \geq \mathfrak{t}_{\omega \gamma+1} \geq \cdots \geq \mathfrak{t}_{\omega \gamma+r} \geq \cdots \geq \omega_{1}$ and, hence, there is $r_{0} \in \omega$ such that $\mathfrak{t}_{\omega \gamma+r}=\mathfrak{t}_{\omega \gamma+r_{0}}$, for each $r \geq r_{0}$.

Proof. (a) By Theorem 1.1, for each $\delta<\omega_{1}$ the poset $\mathrm{sq}\left\langle\mathbb{P}\left(\omega^{\delta}\right), \subset\right\rangle$ is $\omega_{1}$-closed and, by Theorem [5.3. $(P(\omega) / \text { Fin })^{+} \hookrightarrow_{c} \operatorname{sq}\left\langle\mathbb{P}\left(\omega^{\delta}\right), \subset\right\rangle$. Thus $\omega_{1} \leq \mathfrak{t}_{\omega^{\delta}} \leq \mathfrak{h}_{\omega^{\delta}} \leq$ $\mathfrak{h}$. It is known $($ see $[9])$ that $\mathfrak{h}\left((\operatorname{rp}(\mathbb{B}))^{+}\right) \leq \mathfrak{h}\left(\mathbb{B}^{+}\right)$, for each Boolean algebra $\mathbb{B}$ satisfying $\mathfrak{h}\left(\mathbb{B}^{+}\right) \geq \omega_{1}$, so, by (6.1), $\mathfrak{h}_{\omega \gamma+r+1}=\mathfrak{h}\left(\left(\operatorname{rp}^{r+1}\left(P\left(\omega^{\gamma}\right) / \mathcal{I}_{\omega \gamma}\right)\right)^{+}\right)=$ $\mathfrak{h}\left(\left(\operatorname{rp}\left(\operatorname{rp}^{r}\left(P\left(\omega^{\gamma}\right) / \mathcal{I}_{\omega \gamma}\right)\right)\right)^{+}\right) \leq \mathfrak{h}\left(\left(\operatorname{rp}^{r}\left(P\left(\omega^{\gamma}\right) / \mathcal{I}_{\omega^{\gamma}}\right)\right)^{+}\right)=\mathfrak{h}\left(\left(P\left(\omega^{\gamma+r}\right) / \mathcal{I}_{\omega \gamma+r}\right)^{+}\right)=$ $\mathfrak{h}_{\omega \gamma+r}$.

(b) First we prove that $\mathfrak{t}_{\omega \gamma} \leq \mathfrak{t}$, for $\gamma \in$ Lim. By Proposition 4.6. $\left\langle P\left(\omega^{\gamma}\right) \backslash \mathcal{I}_{\omega^{\gamma}}, \subset\right\rangle$ $\cong\langle P(L) \backslash \mathcal{I}, \subset\rangle$ which implies $\left(P\left(\omega^{\gamma}\right) / \mathcal{I}_{\omega \gamma}\right)^{+} \cong(P(L) / \mathcal{I})^{+}$. Thus, by Claim 4.7. $\mathfrak{t}_{\omega \gamma}=\mathfrak{t}\left(\left(P\left(\omega^{\gamma}\right) / \mathcal{I}_{\omega \gamma}\right)^{+}\right)=\mathfrak{t}\left((P(L) / \mathcal{I})^{+}\right)=\mathfrak{t}\left(P(L) \backslash \mathcal{I}, \subset_{\mathcal{I}}\right) \leq \mathfrak{t}$. The rest of the proof is similar to the proof of (a). We use the fact (see 9] ) that $\mathfrak{t}\left((\operatorname{rp}(\mathbb{B}))^{+}\right) \leq \mathfrak{t}\left(\mathbb{B}^{+}\right)$, for each Boolean algebra $\mathbb{B}$ satisfying $\mathfrak{t}\left(\mathbb{B}^{+}\right) \geq \omega_{1}$.

Example 6.2. By Corollary [3.5(a) we have $\mathcal{I}_{\omega^{2}} \cong$ Fin $\times$ Fin and, hence, $\mathfrak{h}_{\omega^{2}}=$ $\mathfrak{h}\left((P(\omega \times \omega) /(\text { Fin } \times \text { Fin }))^{+}\right)$. In [3] Hernández-Hernández proved that in the Mathias model $\mathfrak{h}\left((P(\omega \times \omega) /(\text { Fin } \times \text { Fin }))^{+}\right)=\omega_{1}$, while $\mathfrak{h}=\mathfrak{c}=\omega_{2}$. So, by Theorem 6.1, in this model we have $\omega_{2}=\mathfrak{c}=\mathfrak{h}=\mathfrak{h}_{\omega^{1}}>\mathfrak{h}_{\omega^{2}}=\mathfrak{h}_{\omega^{3}}=\cdots=\omega_{1}$.

By a result of Szymański and Zhou [13] the poset $(P(\omega \times \omega) /(\text { Fin } \times \text { Fin }))^{+}$is not $\omega_{2}$-closed. Thus, by Theorem 6.1(b), $\mathfrak{t}_{\omega^{2}}=\mathfrak{t}_{\omega^{3}}=\cdots=\omega_{1}$ holds in ZFC.

We remark that the generic ultrafilter of $(P(\omega \times \omega) /(\text { Fin } \times \text { Fin }))^{+}$was analyzed by Blass, Dobrinen and Raghavan in [1] and similar analysis may be possible for the other quotient algebras appearing in this paper.

\section{REFERENCES}

[1] A. Blass, N. Dobrinen, D. Raghavan, The next best thing to a P-point, submitted, http://arxiv.org/abs/1308.3790

[2] Roland Fraïssé, Theory of relations, Revised edition, Studies in Logic and the Foundations of Mathematics, vol. 145, North-Holland Publishing Co., Amsterdam, 2000. With an appendix by Norbert Sauer. MR 1808172 (2002d:03084)

[3] Fernando Hernández-Hernández, Distributivity of quotients of countable products of Boolean algebras, Rend. Istit. Mat. Univ. Trieste 41 (2009), 27-33 (2010). MR2676962 (2011j:03113)

[4] Thomas Jech, Set theory, 2nd ed., Perspectives in Mathematical Logic, Springer-Verlag, Berlin, 1997. MR.1492987 (99b:03061)

[5] Kenneth Kunen, Set theory, Studies in Logic and the Foundations of Mathematics, vol. 102, North-Holland Publishing Co., Amsterdam, 1980. An introduction to independence proofs. MR.597342 (82f:03001)

[6] Miloš S. Kurilić and Stevo Todorčević, Forcing by non-scattered sets, Ann. Pure Appl. Logic 163 (2012), no. 9, 1299-1308, DOI 10.1016/j.apal.2012.02.004. MR.2926285

[7] Miloš S. Kurilić, From $A_{1}$ to $D_{5}$ : towards a forcing-related classification of relational structures, J. Symb. Log. 79 (2014), no. 1, 279-295, DOI 10.1017/jsl.2013.26. MR3226025

[8] Miloš S. Kurilić, Posets of copies of countable scattered linear orders, Ann. Pure Appl. Logic 165 (2014), no. 3, 895-912, DOI 10.1016/j.apal.2013.11.005. MR3142392

[9] M. S. Kurilić, Reduced products, submitted.

[10] Richard Laver, On Fraïssé's order type conjecture, Ann. of Math. (2) 93 (1971), 89-111. MR.0279005 (43 \#4731)

[11] Joseph G. Rosenstein, Linear orderings, Pure and Applied Mathematics, vol. 98, Academic Press Inc. [Harcourt Brace Jovanovich Publishers], New York, 1982. MR662564 (84m:06001) 
[12] Saharon Shelah and Otmar Spinas, The distributivity numbers of finite products of $\mathcal{P}(\omega) /$ fin, Fund. Math. 158 (1998), no. 1, 81-93. MR1641157 (2000b:03170)

[13] A. Szymański, Zhou Hao Xua, The behaviour of $\omega^{2^{*}}$ under some consequences of Martin's axiom, General topology and its relations to modern analysis and algebra, V (Prague, 1981), 577-584, Sigma Ser. Pure Math., 3, Heldermann, Berlin, 1983.

Department of Mathematics and Informatics, Faculty of Science, University of Novi Sad, Trg Dositeja Obradovića 4, 21000 Novi Sad, Serbia

E-mail address: milos@dmi.uns.ac.rs 